\title{
Perioperative Management in Patients With Undergoing Direct Oral Anticoagulant Therapy in Oral Surgery - A Multicentric Questionnaire Survey
}

\author{
CLARISSA PRECHT ${ }^{1 *}$, YELIZ DEMIREL ${ }^{1 *}$, ALEXANDRE T. ASSAF ${ }^{1}$, HANS O. PINNSCHMIDT $^{2}$, \\ CHRISTIAN KNIPFER ${ }^{1}$, HENNING HANKEN ${ }^{1}$, REINHARD E. FRIEDRICH ${ }^{1 \#}$ and JOHANNES WIKNER ${ }^{1 \#}$ \\ ${ }^{1}$ Department of Oral and Maxillofacial Surgery, \\ University Medical Center Hamburg-Eppendorf, Hamburg, Germany; \\ ${ }^{2}$ Department of Medical Biometry and Epidemiology, \\ University Medical Center Hamburg-Eppendorf, Hamburg, Germany
}

\begin{abstract}
Aim: The purpose of this study was to survey the current opinions of hospitals and medical practices concerning the perioperative management of patients undergoing direct oral anticoagulant therapy (DOAC) and discuss recommendations for the clinical practice. Materials and Methods: A questionnaire with 13 topics and multiple ordinal-polytomous subitems was designed and sent to 120 Departments of Oral and Maxillofacial Surgery in Austria, Switzerland and Germany, as well as to 85 oral and maxillofacial/oral surgeons in medical offices in Hamburg, Germany. The data were statistically evaluated by Chisquare, Fisher's exact and Jonckheere-Terpstra tests. Results: The rate of response was $42 \%$. Thirty-seven percent of respondents reported treating over 50 patients per year with undergoing DOAC therapy and only $18 \%$ assess a high bleeding risk [33\% for vitamin $K$ antagonists (VKA)]. In contrast to that, $62 \%$ of respondents would interrupt the DOAC therapy for extraction of one tooth, while $94 \%$ would continue VKA therapy. Significantly more clinicians apply suture than those in a medical office. The use of additional hemostatic measures varied between clinic and medical practice. There was a clear request for more detailed guidelines. Conclusion: The study shows the current opinion for perioperative management of patients undergoing DOAC
\end{abstract}

This article is freely accessible online.

*\#These Authors contributed equally to this study.

Correspondence to: Professor Dr. Dr. Reinhard E. Friedrich, University Medical Center Hamburg - Eppendorf, Department of Oral and Maxillofacial Surgery, Martinistraße 52, 20246 Hamburg, Germany. Tel: +49 40741053259, e-mail: r.friedrich@uke.de

Key Words: Postoperative bleeding, direct oral anticoagulant therapy, vitamin $\mathrm{K}$ antagonist, dental surgery. therapy. Multi-centric studies under controlled conditions are needed for a safer treatment of anticoagulated patients as therapy strategies differ greatly between institutions and therefore a complication analysis is hardly possible.

For over 50 years, vitamin $\mathrm{K}$ antagonists (VKAs) are used for systemic anticoagulation in patients with atrial fibrillation, transient ischemic attacks, strokes, pulmonary embolism, venous thrombosis and myocardial infarction (1). Depending on the bleeding risk during surgery there are different recommendations regarding continuation or discontinuation of the VKA therapy (2). Discontinuation is normally concomitant with a bridging therapy using lowmolecular-weight heparin (LMWH) $(3,4)$. In dental surgery, the risk of life-threatening bleeding is low and the site of surgery is accessible, which is why some studies recommend a continuation of VKA therapy (5-7). The risk of thromboembolic events after cessation or bridging of oral anticoagulation is reported to be $0.8 \%$, including $0.2 \%$ fatal events (5). However, discontinuation is still frequently practiced and many patients interrupt their therapy without consulting their medical professionals (8).

Recently, new direct oral anticoagulants (DOACs) were introduced to widen the field of anticoagulation $(1,9)$. In large systematic studies it has been reported that dabigatran, rivaroxaban, apixaban or edoxaban show comparable, or even less hemorrhagic side-effects than VKAs and are regarded as a feasible alternative for oral anticoagulation $(10$, 11). Advantageous is the short half-life of these drugs (dabigatran: 12-17 hours; rivaroxaban: 5-13 hours; apixaban: 12-18 hours) (12). However, there are different recommendations regarding the perioperative management of DOAC therapy in dental surgery, such as either pausing DOAC intake for 24 hours prior to surgery with low bleeding risk and continuing 24 to 48 hours afterwards, or waiting for 4 to 6 hours after the last intake when conducting 
surgery or no pause of intake at all $(9,13)$. Studies report that local hemostasis seems to be sufficient to prevent postoperative bleeding (9). However, there are no recommendations regarding perioperative management for edoxaban, the newest member of the DOAC family (14). Empirical-based practical guidelines of how to handle DOAC therapy in dental surgery are non-existent and large clinical studies are still lacking (14-18). Which is why the clinical pathway tends to be different for each institution. A representative opinion poll regarding empirical knowledge could be helpful at this point. Therefore, the aim of this multicentric questionnaire survey was to gain insight into the current perioperative management of patients treated with DOACs at different clinical institutions and medical practices located in Germany, Austria and Switzerland.

\section{Materials and Methods}

Questionnaire. A German paper-based questionnaire concerning the perioperative management of patients undergoing DOAC, VKA or antiplatelet therapy was designed. The questionnaire covered 13 topics and multiple ordinal-polytomous subitems concerning the estimated risk of bleeding, different presurgical and surgical approaches depending on the clinical situation, the necessity for laboratory tests, the use of additional hemostatic measures and a request for more detailed guidelines from clinical associations. General information was recorded in the first part of the questionnaire. The questions included the kind of the facility (clinical institution or medical practice), the profession of the respondent (oral surgeon or oral and maxillofacial surgeon) and the number of treated patients who received coagulation inhibitors.

At this point, a distinction was made between VKA, acetylsalicylic acid (ASA)/clopidogrel (antiplatelet therapy), DOAC and bridging with LMWH. In this context, the respondents provided information about the frequency of postoperative complications and the necessity of inpatient treatment. In comparison to healthy patients, the bleeding risk was ranked on a scale from unchanged to ++++ (+: low, ++: moderate, +++: high, ++++: extremely high).

The second part of the questionnaire focused on the individual's opinion concerning the occurrence of postoperative bleeding comparing healthy patients and patients receiving one of the aforementioned coagulation inhibitors. The respondents answered whether in the case of different surgical treatments, such as extraction or osteotomy of one tooth, the extraction of more teeth or complicated osteotomies, they preferred to maintain the anticoagulant therapy, to pause it, or to bridge with LMWH. The participants were asked about the necessity for laboratory tests and their timing before surgery. Furthermore, the respondents evaluated whether the manufacturer's instructions for use were sufficient and if they would request more studies and more detailed guidelines for perioperative management of patients undergoing DOAC therapy.

The third part of the questionnaire included an example case. The respondents were asked about the frequency of additional hemostatic measures they would take in the case of extraction of 35 teeth under compromised coagulation. The measures were predetermined as "non-surgical measures", "wound closure with stitches", "slotted/non-slotted mucoperiosteal flap", "bolting", "electrocautery", "use of fibrin adhesive", "use of oxicellulose", "use of gingiva bandage", "use of desmopressin", "use of tranexamic acid" and "use of a cradle", and were ranked with "never", "rarely", "moderate", "frequently" and "very frequently".

The last part of the questionnaire asked about the influence of different parameters which might increase the probability of potential bleeding, rating them from insignificant to significant on a scale from 1 (insignificant) to 5 (significant).

Participants. The questionnaire was sent with a request to complete and return it to 120 Departments of Oral and Maxillofacial Surgery in Austria, Switzerland and Germany as well as to 85 oral and maxillofacial and oral surgeons at medical offices in Hamburg, Germany during the years 2016 to 2017 .

Statistical evaluation. Relationships between categorical variables were examined by chi-square and Fisher's exact tests. Directional relationships amongst dichotomous, ordinal and count variables were examined by Jonckheere-Terpstra tests. Scores indicating how often surgical and non-surgical measures were performed after extraction and scores indicating opinions about bleeding risk factors are presented as means and $95 \%$ confidence intervals. The software package SPSS 24 (IBM, Armonk, New York, USA) was used for all statistical analyses. The two-tailed level of significance was set at $p<0.05$

\section{Results}

Out of 120 mailed requests, 56 Departments of Oral and Maxillofacial Surgery participated in this questionnaire survey (47\%). The respondents were mainly from Germany (93\%), departments from Austria and Switzerland seldom responded (6\% and 1\%). In Hamburg (Germany), 85 oral and maxillofacial and oral surgeons in medical practices were contacted and 30 responded $(35 \%)$.

Sixty-two participants $(78 \%)$ reported treating more than 50 patients per year with a dual anticoagulation therapy with ASA/clopidogrel. There was no significant difference comparing clinics and medical practices $(p=0.417)$ : 59 participants $(70.2 \%)$ treated more than 50 patients who received anticoagulation therapy with VKAs per year. Nineteen percent saw 20-50 such patients per year. Significantly more patients were treated in clinical departments than in medical practices $(p<0.0001)$. Thirty-nine percent of the participants reported that they treated more than 50 patients per year with LMWH therapy. Significantly more of these patients were treated in a hospital $(p<0.0001)$. DOAC therapy was less common: $27 \%$ of the institutions treated $20-50$ patients and $37 \%$ treated more than 50 patients per year. The difference between hospital and medical practice was significant in favor of hospitals $(p=0.013)$.

About 56\% of the surgeons specified that fewer than 5\% of their patients receiving VKAs needed inpatient treatment after surgery. Significantly fewer patients needed stationary treatment after surgery in medical practices than in hospitals $(p<0.001)$. A similar proportion $(54 \%)$ reported the same for LMWH-treated patients and even $71.6 \%$ for dual 


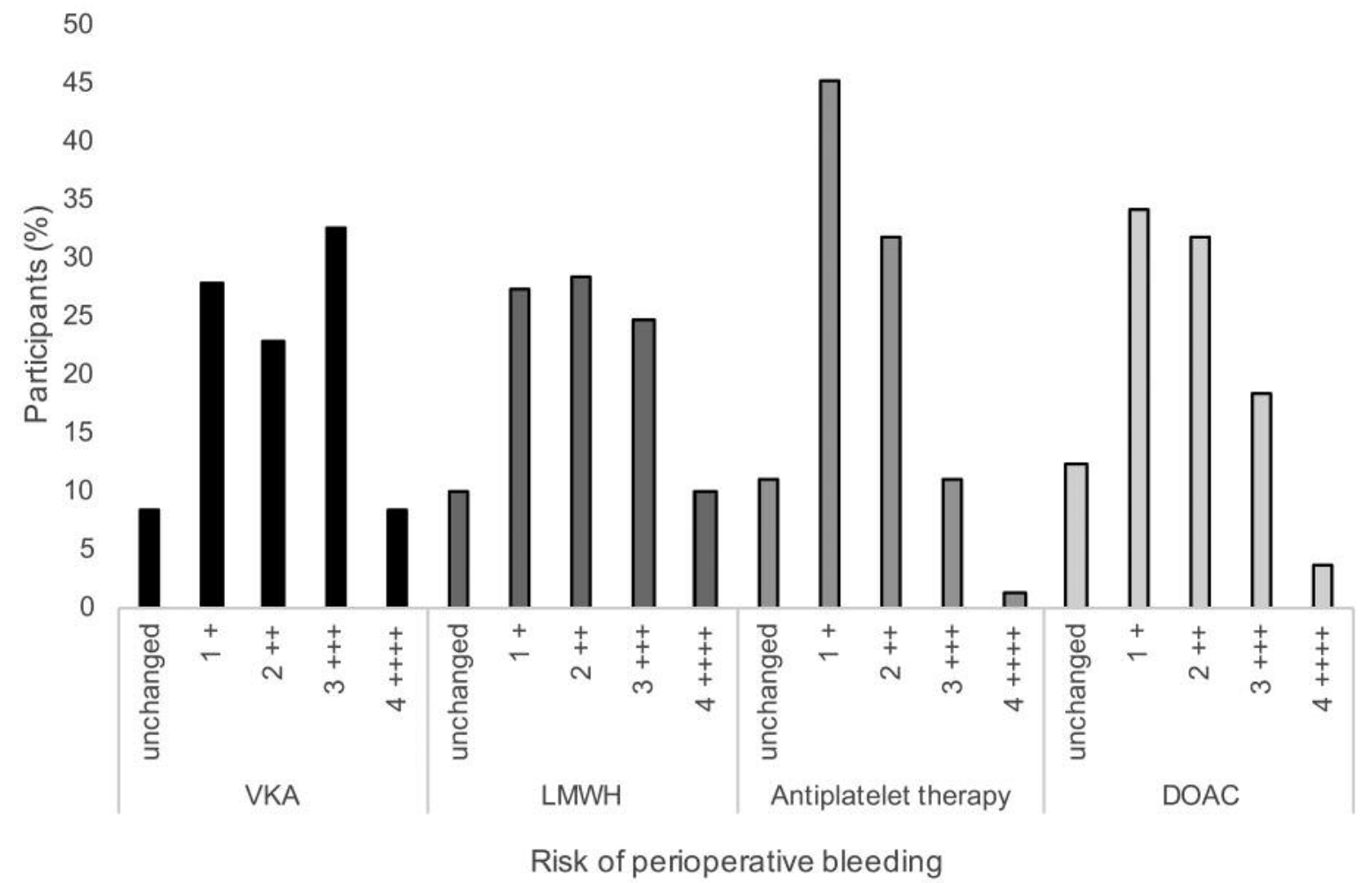

Figure 1. Risk of bleeding after dental surgery in patients treated with vitamin K antagonist (VKA), low-molecular-weight heparin (LMWH) bridging, antiplatelet therapy, or direct oral anticoagulant $(D O A C)$ as rated by study participants. Risk scale: $+:$ low, $++:$ moderate, $+++:$ high, ++++ : extremely high.

anticoagulation therapy with ASA/clopidogrel $(p<0.001$; $p=0.001)$. DOAC therapy was also in this range: $68.4 \%$ of the surgeons stated that fewer than $5 \%$ of their patients receiving DOACs needed inpatient treatment. Significantly fewer patients needed inpatient treatment after surgery in medical practices than in hospitals $(p<0.001)$.

The rate of severe complications for VKAs was described as rare by $61 \%$ of the participants $(p=0.382)$, for LMWH by $54.2 \%$ ( $p=0.001$, in favor of medical practices), for dual anticoagulation therapy with ASA/clopidogrel by $61.9 \%$ $(p=0.697)$ and for DOAC therapy by $63.9 \%(p=0.631)$.

In comparison to healthy patients, the risk of perioperative bleeding was considered on a scale from "unchanged" to high $(++++)$ (Figure 1). The bleeding risk for DOAC was estimated to be lower in comparison to that for VKAs. Among all participants, $32.5 \%$ ranked the perioperative bleeding risk for VKA with +++ in comparison to only $18 \%$ for those undergoing DOAC therapy. There was no significant difference between hospitals and medical practices in this regard $(p=0.975)$. The participants ranked the bleeding risk for patients treated with LMWH higher than that for therapy with ASA/clopidogrel (9.9\% versus $1.2 \%$ as,$++++ 24.7 \%$ and $11 \%$ as +++$)$. There was a significant difference for the ranking of bleeding risk after surgery for those undergoing LMWH therapy between hospitals and medicals practice: the respondents in medical practice ranked the bleeding risk significantly lower than did the clinicians $(p=0.012)$.

In the opinion of most of the respondents, laboratory tests were not considered necessary for patients treated with ASA/clopidogrel (78\%, $p=0.801)$ and DOACs $(67 \%$; $p=0.806)$. Twenty-one percent supposed that tests should be performed for patients treated with DOACs before surgery and $10 \%$ directly at the time of surgery. In contrast to ASA/clopidogrel and DOACs, the conducting of laboratory tests was rated as more important for those treated with VKA. Forty-four percent stated they would perform tests in patients who received VKAs before surgery and $29 \%$ directly at the time of surgery; only $4 \%$ deemed laboratory tests unnecessary $(p=0.505)$. For LMWH, 32\% said they would perform laboratory tests before an operation and $23 \%$ directly at the time of operation, but $30 \%$ did not see a need for laboratory tests $(p=0.117)$.

Most of the respondents stated that they would perform interventions such as extraction of one tooth $(94 \%, p=0.522)$ or of two to five teeth (84\%) and an osteotomy of a tooth 


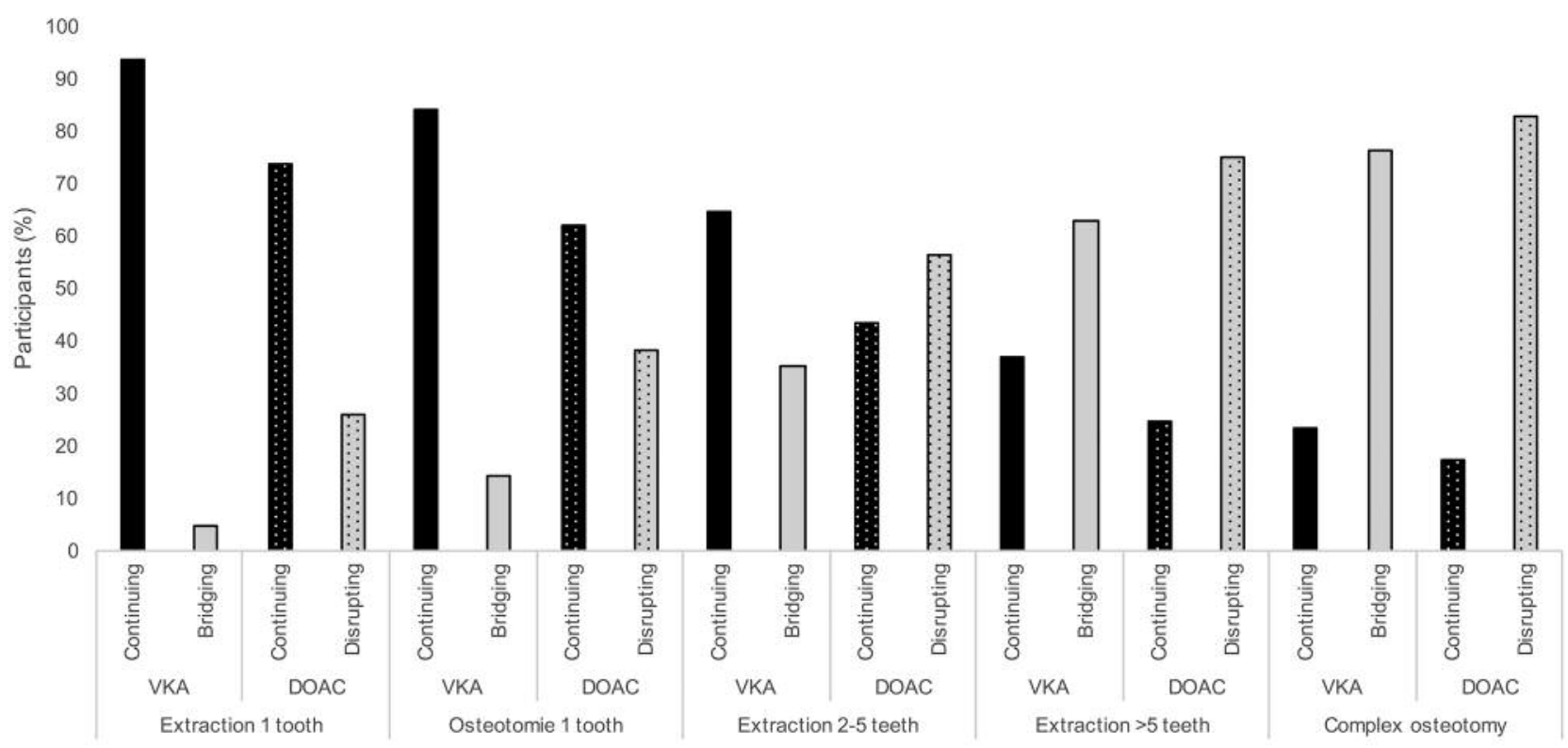

Figure 2. Percentage of respondents who stated they would continue or bridge vitamin K antagonist therapy (VKA) or continue or disrupt (according to manufacturer's guidelines or medical professional's recommendations) direct oral anticoagulant (DOAC) therapy when undertaking extraction of one tooth, osteotomy of one tooth, extraction of 2-5 teeth, extraction of more than 5 teeth, or complex osteotomy.

(65\%) while continuing VKA therapy. Clinicians would continue VKA therapy more often, when two to five teeth were to be extracted $(p=0.046)$ or an osteotomy was needed $(p<0.0001)$, than surgeons in medical practices. For the extraction of more than five teeth $(63 \%, p=0.091)$ and complicated osteotomies $(77 \%, p=0.56)$, they preferred to bridge their patients with LMWH. In comparison to therapy with VKAs, the approach during DOAC therapy was found to be more cautious: $57 \%$ of participants stated they would discontinue DOAC therapy for an extraction of two to five teeth and would consult an internist $(p=0.159)$. Seventy-five percent said would discontinue the therapy for extraction of more than five teeth $(p=0.169)$ and $83 \%$ for complicated osteotomies $(p=0.119)$. In contrast to that, $73.8 \%$ stated they would continue the therapy for extraction of one tooth $(\mathrm{p}>0.99)$ and $61.9 \%$ for an osteotomy of one tooth $(p=0.104)$ (Figure 2).

This survey showed that only $64.5 \%$ of the participants regarded the producer's recommendations for dental-surgical interventions as sufficient. The majority (94\%) saw a need for further studies related to DOAC treatment. Standardized laboratory tests were requested from $71 \%$ of the respondents, and $98 \%$ wished for the formulation of guidelines for safe handling of patients treated with DOACs.

The participants were asked about their approach to an extraction of three to five teeth in patients with compromised coagulation (Figure 3). Sixty percent did not regard nonsurgical local measures, such as curettage and the use of gauzes, as necessary. The difference between clinical institutions and medical practices was not statistically significant $(p=0.438)$. In contrast, the majority $(74 \%)$ preferred wound closure with stitches, with a statistically significant difference in favor of the clinicians $(p=0.005)$. Different measures of flap design were selected. The nonslotted mucoperiosteal flap was used rarely by $25 \%$, moderately by $29 \%$, and frequently by $28 \%$. The rates for slotted mucoperiosteal flaps were different ( $22 \%$ moderately, $16 \%$ frequently and $10 \%$ very frequently). There were no significant differences for both flaps comparing clinic and medical practices ( $p=0.170$ and $p=0.088$, respectively). Electrocautery was used significantly more often in the clinic than in medical practices $(p=0.014)$. Bolting was used rarely in clinic and medical practices $(p=0.130)$. Several respondents used local measures such as fibrin adhesive (44\% never and $44 \%$ rarely), gingiva bandage (35\% never and $39 \%$ rarely) and desmopressin (38\% never and $51 \%$ rarely) never or rarely. Comparing the institutions, only desmopressin was found to be used significantly more often in the clinic than in medical practices $(p=0.019)$. Compared to the clinic, tranexamic acid was not widely used in medical practices $(p<0.0001)$. Ninety-eight percent of the medical practices never or only seldomly used tranexamic acid. There was no difference in the use of oxicellulose (Tabotamp ${ }^{\mathrm{TM}}$; Ethicon, Johnson \& Johnson Medical GmbH, USA): $31.25 \%$ used oxicellulose very frequently. Cradles were never customized in $24 \%$ of the cases, customized moderately 


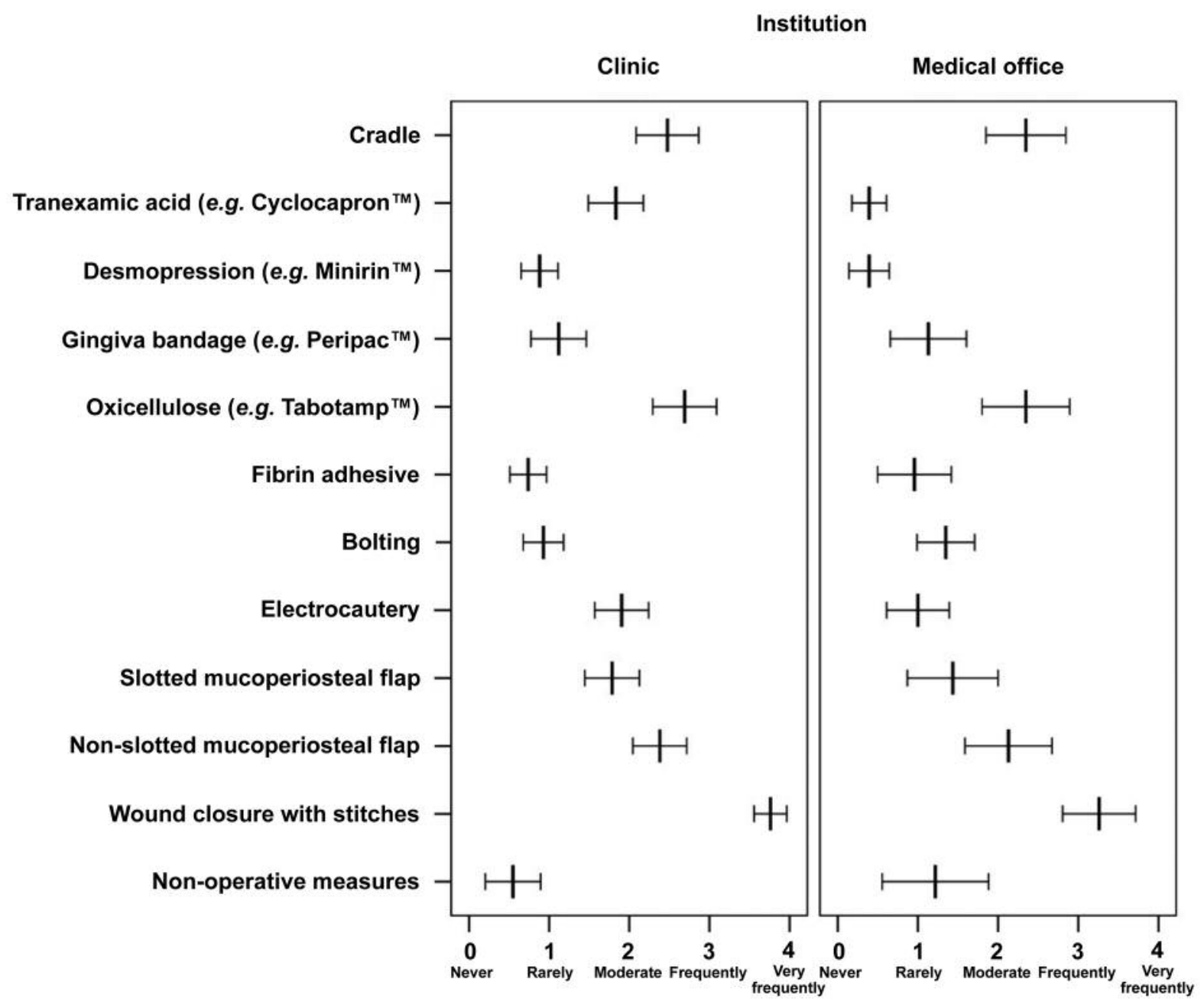

Figure 3. Use of different surgical and non-surgical measures ranked from 0 (never) to 4 (very frequently) in the scenario of a patient under anticoagulation therapy after extraction of 3-5 teeth comparing respondents from clinics and medical offices. Mean frequency scores and their $95 \%$ confidence intervals are shown.

often in $27 \%$, in $20 \%$ frequently and in $24 \%$ very frequently; there was no significant difference between clinics and medical practices $(p=0.821)$.

Factors such as blood pressure, localization of the defect in the front/canine region suture, suture material used, tightness of the suture, curettage of the socket and composition of the saliva were ranked as 1 (insignificant) or 2 (nearly insignificant) for the risk of bleeding (Figure 4) Osseous defect, the length of operation, localization of the defect at the lower molar, localization of the defect at the upper molar and use of mucoperiosteal flap were rated as a 3 . The experience of the surgeon, high blood pressure and the necessity for periosteal incision were ranked as a 4 . None of the factors were significantly differently ranked between hospitals and medical offices, except for infection: surgeons in the clinic rated this factor mostly with a 3 and to a lesser extent with a 4, whereas surgeons in medical practices estimate it to have a higher importance of 4 and even with 5 $(p=0.006)$.

\section{Discussion}

In this study, we conducted an opinion poll regarding the perioperative management of patients with DOAC therapy by sending questionnaires to clinical institutions and medical practices located in Germany, Austria and Switzerland. To our knowledge this is the first study which gathered expert opinions regarding dental surgery in patients undergoing DOAC therapy.

Patients who receive therapy with DOACs are becoming more frequent in daily practice. In our study, DOAC therapy was still less common than VKA therapy, but nevertheless $27 \%$ 


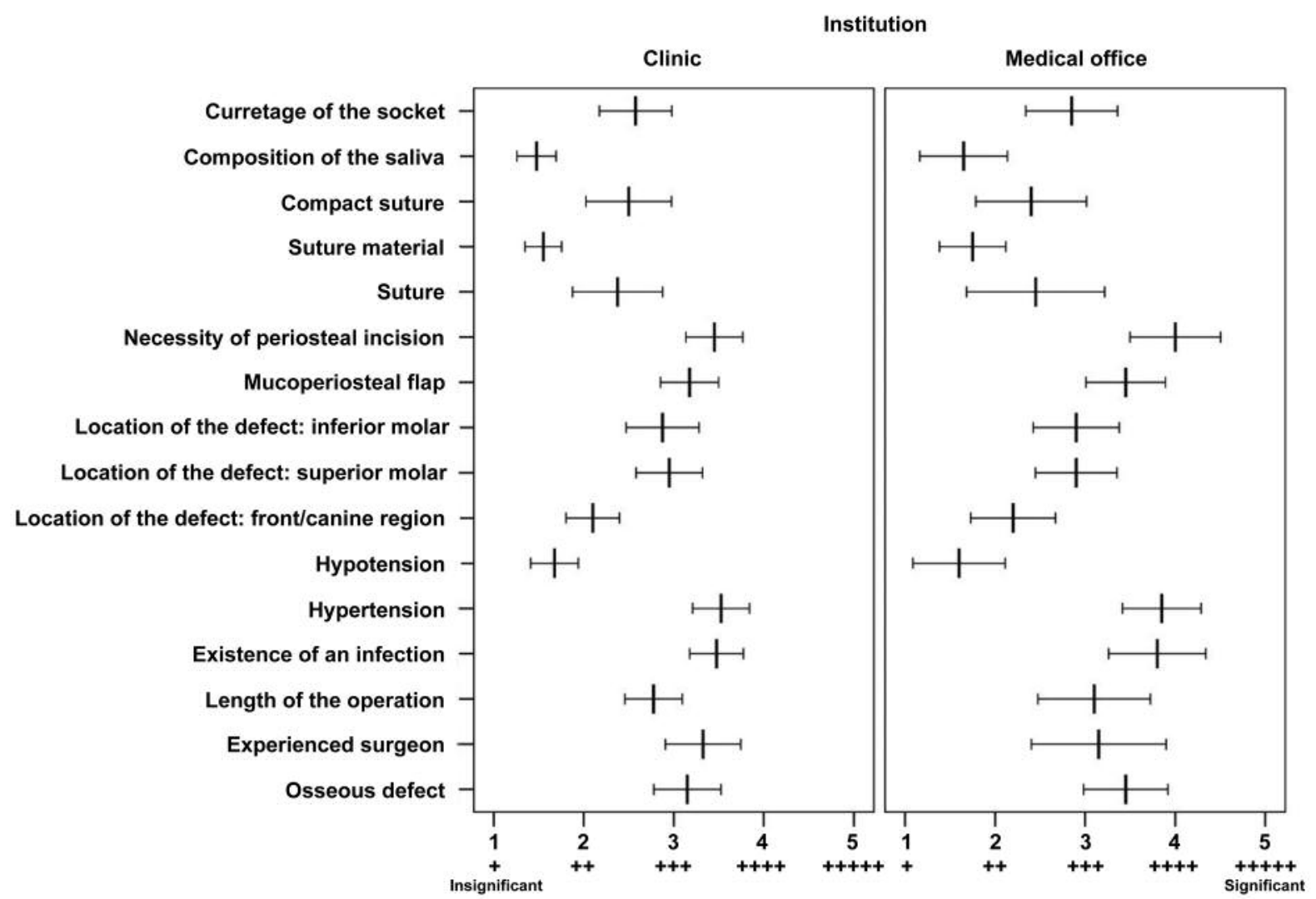

Figure 4. Evaluation of factors considered to increase bleeding risk ranked in the scenario of a patient under anticoagulation from 1 (+, insignificant) to $5(+++++$, significant) by respondents from clinics and medical offices. Mean factor scores and their $95 \%$ confidence intervals are shown.

of the institutions treated $20-50$ patients and $37 \%$ treated more than 50 patients per year. Most patients treated with DOACs were seen in hospitals, but inpatient treatment was needed less frequently after dental surgery for those undergoing DOAC therapy than those undergoing VKA therapy; $68.4 \%$ of the surgeons stated that fewer than $5 \%$ of their patients receiving DOACs needed stationary treatment; only $56 \%$ did the same for VKA therapy. This was conclusive as the respondents' estimated bleeding risk in comparison with patients not anticoagulant was lower for those on DOACs than for those on VKAs. This is in line with the findings of Clemm et al., who reported a higher bleeding rate for patients treated with VKAs than with DOACs (19). Although Hanken et al. found a bleeding rate of $11.5 \%$ after minor oral surgery in patients undergoing rivaroxaban therapy (20). Interestingly, 34.6\% of the participants of our study ranked the bleeding risk of LMWH therapy as very high or high. This is in line with reports in the literature. BeyerWestendorf et al. found that the prevalence of bleeding was higher in a group of patients who received LMWH as bridging (21). In our study, severe complications were considered rare for VKA, DOAC or antiplatelet therapy. This rate was estimated a little higher for LMWH.
When planning a surgical measure, VKA therapy is often interrupted; patients with a high thromboembolic risk are bridged with LMWH in fear of hemorrhagic complications (3, 4). Several studies stated that dental surgery has a low risk of bleeding and the mouth is easily accessible for local hemostatic measures, such as wound closure with suture, gelatin sponge, fibrin adhesive, oxicellulose, tranexamic acid or cradles $(5,12)$. They found a higher thromboembolic risk during interruption of VKA therapy, which is why some investigators recommended not disrupting anticoagulation for interventions with low bleeding risk $(5-7,16)$. However, studies concerning handling of DOAC treatment in dental surgery are small in number, as well as in size and diversity. Convincing large clinical studies are still lacking (14-18). For instance, Breik et al. published a management protocol derived from a literature review and their own experience and stated that while single tooth extractions are feasible without discontinuation of dabigatran, disruption should be discussed with the physician when multiple extractions are planned. The authors suggested restarting the treatment 24 to 48 hours postoperatively (22). Timing of discontinuation is dependent on elimination half-life and renal function (12). Van Diermen et al. stated that patients 
should not take their medication between 1 and 3 hours prior to the extraction of up to three teeth (6), which is in line with a report from Syyed et al., in which they recommended continuation of dabigatran when undergoing extraction of up to three teeth (23). A review article from Nathwani et al. compared three studies $(14,24,25)$ and expert-formed guidelines from the Scottish Dental Clinical Effectiveness Programme and concluded that dental surgery in patients taking DOACs can either be done without discontinuation of therapy or a delay in dose. Contacting a specialist physician is necessary when risk of bleeding is high (15). In our study, we asked the participants if they would continue or disrupt anticoagulation with VKAs or DOACs when facing certain scenarios. Most surgeons stated they would continue VKA or DOAC therapy when performing a single tooth extraction or an osteotomy of one tooth. Comparing the two treatments, the approach during DOAC therapy was more cautious concerning extraction of two to five teeth. While most participants stated they would continue VKA therapy, the majority said they would discontinue DOAC therapy. Moreover, clinicians more often stated they would continue VKA therapy than did surgeons in medical practices. For the extraction of more than five teeth and complicated osteotomies, they preferred to disrupt both treatments. These results are interesting as the participants answered earlier that they saw fewer bleeding complications in patients treated with DOACs than in patients taking VKAs. Ward and Smith, in their study defining international normalized ratio (INR) cut-offs during VKA therapy for performing certain surgical procedures, showed that the extent of the procedure significantly influenced the individual anticoagulation management of the surgeon (26). Interestingly, clinicians more often applied suture to extraction wounds than participants in medical offices. This is likely due to the costs of suture material and the extended time needed for suturing. Furthermore, the clinics are available as back-up in case of a bleeding event.

VKA activity can be tested by measuring the INR in blood specimens and oral surgery can be conducted in the normal therapeutic ranges of an INR between 2 to $3(5,12,16)$. Standardized laboratory tests for DOAC activity were requested in our study from $71 \%$ of the respondents, but to date, tests are rarely helpful as they are not sensitive enough or not widely available $(12,18)$. Considering the lack of opportunity for monitoring DOAC activity and the fact that reversal agents are still in a test phase $(12,27)$, the need for empirical-based practical guidelines for treating patients undergoing DOAC therapy in dental surgery is obvious.

\section{Conclusion}

In this multicentric questionnaire survey, we gained a valuable insight into the diversity of perioperative management strategies of patients under coagulation therapy in Germany, Austria and Switzerland. The postoperative bleeding risk seems to be equivalent comparing VKAs and the new DOACs, but surgeons are still more cautious conducting dental surgery in patients undergoing DOAC therapy. Empirical-based practical guidelines for dental surgery in patients treated with DOACs are still lacking, but the need is obvious.

\section{Conflicts of Interest}

The Authors declare no conflicts of interest in regard to this study.

\section{Authors' Contributions}

CP, YD, JW: Conception of the study, designed study, collected data, drafting, analyzed data, revised article, final approval, agreement to be accountable for all aspects of the work; HP, RF, AA, HH, CK: interpretation of data, revised article, final approval, agreement to be accountable for all aspects of the work.

\section{Acknowledgements}

This research did not receive any specific grant from funding agencies in the public, commercial, or not-for-profit sectors.

\section{References}

1 Miranda M, Martinez LS, Franco R, Forte V, Barlattani A Jr. and Bollero P: Differences between warfarin and new oral anticoagulants in dental clinical practice. Oral Implantol 9(3): 151156, 2016. PMID: 28042443. DOI: 10.11138/orl/2016.9.3.151

2 van Diermen DE, Aartman IH, Baart JA, Hoogstraten J and van der Waal I: Dental management of patients using antithrombotic drugs: Critical appraisal of existing guidelines. Oral Surg Oral Med Oral Pathol Oral Radiol Endod 107(5): 616-624, 2009. PMID: 19426918. DOI: 10.1016/j.tripleo.2009.01.038

3 Pettinger TK and Owens CT: Use of low-molecular-weight heparin during dental extractions in a medicaid population. $\mathrm{J}$ Manag Care Pharm 13(1): 53-58, 2007. PMID: 17269837. DOI: 10.18553/jmcp.2007.13.1.53

4 Johnson-Leong C and Rada RE: The use of low-molecularweight heparins in outpatient oral surgery for patients receiving anticoagulation therapy. J Am Dent Assoc 133(8): 1083-1087, 2002. PMID: 12198988

5 Wahl MJ, Pinto A, Kilham J and Lalla RV: Dental surgery in anticoagulated patients - stop the interruption. Oral Surg Oral Med Oral Pathol Oral Radiol 119(2): 136-157, 2015. PMID: 25577414. DOI: 10.1016/j.0ooo.2014.10.011

6 van Diermen DE, van der Waal I and Hoogstraten J: Management recommendations for invasive dental treatment in patients using oral antithrombotic medication, including novel oral anticoagulants. Oral Surg Oral Med Oral Pathol Oral Radiol 116(6): 709-716, 2013. PMID: 24120910. DOI: 10.1016/ j.0ooo.2013.07.026

7 Perry DJ, Noakes TJ and Helliwell PS: Guidelines for the management of patients on oral anticoagulants requiring dental surgery. Br Dent J 203(7): 389-393, 2007. PMID: 17934422. DOI: $10.1038 /$ bdj.2007.892 
8 Hanken H, Tieck F, Kluwe L, Smeets R, Heiland M, Precht C, Eichhorn $\mathrm{M}$ and Eichhorn W: Lack of evidence for increased postoperative bleeding risk for dental osteotomy with continued aspirin therapy. Oral Surg Oral Med Oral Pathol Oral Radiol 119(1): 17-19, 2015. PMID: 25442244. DOI: 10.1016/ j.oooo.2014.08.016

9 Mauprivez C, Khonsari RH, Razouk O, Goudot P, Lesclous P and Descroix V: Management of dental extraction in patients undergoing anticoagulant oral direct treatment: A pilot study. Oral Surg Oral Med Oral Pathol Oral Radiol 122(5): e146-e155, 2016. PMID: 27554378. DOI: 10.1016/j.oooo.2016.06.003

10 Patel MR, Mahaffey KW, Garg J, Pan G, Singer DE, Hacke W, Breithardt G, Halperin JL, Hankey GJ, Piccini JP, Becker RC, Nessel CC, Paolini JF, Berkowitz SD, Fox KA and Califf RM: Rivaroxaban versus warfarin in nonvalvular atrial fibrillation. $\mathrm{N}$ Engl J Med 365(10): 883-891, 2011. PMID: 21830957. DOI: 10.1056/NEJMoa1009638

11 Granger CB, Alexander JH, McMurray JJ, Lopes RD, Hylek EM, Hanna M, Al-Khalidi HR, Ansell J, Atar D, Avezum A, Bahit MC, Diaz R, Easton JD, Ezekowitz JA, Flaker G, Garcia D, Geraldes M, Gersh BJ, Golitsyn S, Goto S, Hermosillo AG, Hohnloser SH, Horowitz J, Mohan P, Jansky P, Lewis BS, Lopez-Sendon JL, Pais P, Parkhomenko A, Verheugt FW, Zhu J and Wallentin L: Apixaban versus warfarin in patients with atrial fibrillation. N Engl J Med 365(11): 981-992, 2011. PMID: 21870978. DOI: 10.1056/NEJMoa1107039

12 Yurttas T, Wanner PM and Filipovic M: Perioperative management of antithrombotic therapies. Curr Opin Anaesthesiol, 2017. PMID: 28509773. DOI: 10.1097/aco.00000 00000000481

13 Dubois V, Dincq AS, Douxfils J, Ickx B, Samama CM, Dogne JM, Gourdin M, Chatelain B, Mullier F and Lessire S: Perioperative management of patients on direct oral anticoagulants. Thromb J 15: 14, 2017. PMID: 28515674. DOI: 10.1186/s12959-017-0137-1

14 Costantinides F, Rizzo R, Pascazio L and Maglione M: Managing patients taking novel oral anticoagulants (NOAS) in dentistry: A discussion paper on clinical implications. BMC Oral Health 16: 5, 2016. PMID: 26822674. DOI: 10.1186/s12903016-0170-7

15 Nathwani $S$ and Wanis $C$ : Novel oral anticoagulants and exodontia: The evidence. Br Dent J 222(8): 623-628, 2017. PMID: 28428567. DOI: 10.1038/sj.bdj.2017.364

16 Kammerer PW, Frerich B, Liese J, Schiegnitz E and Al-Nawas B: Oral surgery during therapy with anticoagulants-a systematic review. Clin Oral Investig 19(2): 171-180, 2015. PMID: 25413495. DOI: 10.1007/s00784-014-1366-3

17 Miclotte I, Vanhaverbeke M, Agbaje JO, Legrand P, Vanassche $\mathrm{T}$, Verhamme $\mathrm{P}$ and Politis C: Pragmatic approach to manage new oral anticoagulants in patients undergoing dental extractions: A prospective case-control study. Clin Oral Investig 21(7): 2183-2188, 2017. PMID: 27891570. DOI: 10.1007/ s00784-016-2010-1
18 Johnston S: An evidence summary of the management of patients taking direct oral anticoagulants (DOACS) undergoing dental surgery. Int J Oral Maxillofac Surg 45(5): 618-630, 2016. PMID: 26774397. DOI: 10.1016/j.ijom.2015.12.010

19 Clemm R, Neukam FW, Rusche B, Bauersachs A, Musazada S and Schmitt CM: Management of anticoagulated patients in implant therapy: A clinical comparative study. Clin Oral Implants Res 27(10): 1274-1282, 2016. PMID: 26592859. DOI: $10.1111 / \mathrm{clr} .12732$

20 Hanken H, Grobe A, Heiland M, Smeets R, Kluwe L, Wikner J, Koehnke R, Al-Dam A and Eichhorn W: Postoperative bleeding risk for oral surgery under continued rivaroxaban anticoagulant therapy. Clin Oral Investig 20(6): 1279-1282, 2016. PMID: 26498769. DOI: $10.1007 / \mathrm{s} 00784-015-1627-9$

21 Beyer-Westendorf J, Gelbricht V, Forster K, Ebertz F, Kohler C, Werth S, Kuhlisch E, Stange T, Thieme C, Daschkow K and Weiss N: Peri-interventional management of novel oral anticoagulants in daily care: Results from the prospective Dresden NOAC registry. Eur Heart J 35(28): 1888-1896, 2014. PMID: 24394381. DOI: 10.1093/eurheartj/eht557

22 Breik O, Cheng A, Sambrook P and Goss A: Protocol in managing oral surgical patients taking dabigatran. Aust Dent $\mathrm{J}$ 59(3): 296-301; quiz 401, 2014. PMID: 24889878. DOI: 10.1111/adj.12199

23 Syyed N, Ansell M and Sood V: Dabigatran Pradaxa(®): Surgeon's friend or foe? Br Dent J 217(11): 623-626, 2014. PMID: 25476635. DOI:10.1038/sj.bdj.2014.1051

24 Curtin C, Hayes JM and Hayes J: Dental implications of new oral anticoagulants for atrial fibrillation. Dent Update 41(6): 526-528, 530-521, 2014. PMID: 25195485. DOI: 10.12968/ denu. 2014.41 .6 .526

25 Davis C, Robertson C, Shivakumar S and Lee M: Implications of dabigatran, a direct thrombin inhibitor, for oral surgery practice. J Can Dent Assoc 79: d74, 2013. PMID: 23920075.

26 Ward BB and Smith MH: Dentoalveolar procedures for the anticoagulated patient: Literature recommendations versus current practice. J Oral Maxillofac Surg 65(8): 1454-1460, 2007. PMID: 17656268. DOI: 10.1016/j.joms.2007.03.003

27 Deville L, Konan M, Hij A, Goldwirt L, Peyrony O, Fieux F, Faure P, Madelaine I, Villiers S, Farge-Bancel D and Frere C: Major bleeding complications in patients treated with direct oral anticoagulants: One-year observational study in a Paris hospital. Curr Res Transl Med 64(3): 129-133, 2016. PMID: 27765272. DOI: $10.1016 /$ j.retram.2016.02.003 